\title{
Perceptions of older adults and health professionals about digital screening tools for elder mistreatment in the emergency department
}

\author{
Esther K. Choo MD MPH ${ }^{a}$, Chelsea Edwards BS BA ${ }^{b}$, Malik Abuwandi ${ }^{\mathrm{c}}$, Kristina \\ Carlson $^{b}$, Jennifer Bonito ${ }^{b}$, Karen Jubanyik $M^{b}{ }^{b}$, Thomas M. Gill MD ${ }^{d}$, Fuad \\ Abujarad MSc PhD ${ }^{\mathrm{b}, *}$ \\ ${ }^{a}$ Center for Policy \& Research in Emergency Medicine, Oregon Health \& Science Univer- \\ sity, Portland, OR, USA; ${ }^{b}$ Department of Emergency Medicine, Yale School of Medicine, \\ New Haven, CT, USA; ' Arizona State University, Phoenix, AZ, USA; ${ }^{d}$ Department of In- \\ ternal Medicine, Yale School of Medicine, New Haven, CT, USA; *Corresponding author: \\ fuad.abujarad@yale.edu
}

\begin{abstract}
Background: Healthcare settings represent a missed opportunity to systematically identify and address mistreatment.

Objective: Our objective was to obtain perspectives of older adults, caregivers, and emergency care providers regarding screening and intervention for elder mistreatment in the emergency department (ED) with a focus on utilizing digital health tools to facilitate the process. These findings will inform the development of a Web-based, digital health tool optimized for a tablet device to educate, screen, and facilitate reporting of elder mistreatment among patients presenting to the ED.

Method: We conducted a qualitative study utilizing three in-person focus groups $(\mathrm{N}=31)$ with older adults from the community, caregivers for older adults, and clinicians and social workers who worked in the ED. Using a semi-structured interview guide, we identified attitudes about the process of divulging abuse, attitudes towards the ED as the location for screening and information delivery, and perceptions of digital tools for screening and information.

Results: Participants identified numerous challenges to the disclosure of mistreatment, including feelings of vulnerability and concerns about losing their homes, social supports, and connection to caregivers. In contrast, they were uncertain about the benefits of disclosure. Digital tools were seen as helpful in terms of overcoming numerous challenges to screening, but participants suggested maintaining a human element to interactions. Conclusion: While challenges to elder mistreatment screening were identified, participants had recommendations for optimizing such efforts and responded positively to digital health tools as a means of screening.
\end{abstract}

Keywords: elder mistreatment, emergency care, digital health, technology, elder abuse screening

\section{INTRODUCTION}

Elder mistreatment - defined by the Centers for Disease Control and Prevention (CDC) as the intentional act, or failure to act, by a caregiver or trusted person that causes or creates a risk of harm to an adult age 60 or older (Centers for Disease Control and Prevention, 2016). - is a persistent public health challenge. Common forms of elder mistreatment can include physical, emotional, financial, and sexual abuse, and neglect (Storey, 2020). Estimates of the percentage of older adults who experience mistreatment vary widely but may be as high as one in ten (Rosay et al., 2017), although identifying cases consistently for accurate estimates is challenging (Yon et al., 2017; Centers for Disease Control and Prevention, 2016). Over time, the urgency of addressing elder mistreatment will become even more acute: the over- 65 population has grown by over a third in the past decade (United States Census Bureau, 2020). By 2060, nearly a quarter of the U.S. population will be 65 years or older (United States Census Bureau, 2020). Improving systems and services to ensure the safety and quality of life of this vulnerable population is vital to the health and well-being of our nation.

The emergency care visit is a unique opportunity to identify these problems and provide interventions or referrals. Twenty-two percent of adults 65 or older in the U.S. will visit an emergency department (ED) in a given year (Centers for Disease Control and Prevention, 2018). The ED visit has been described as a time in which people may be more inclined to discuss a wide variety of sensitive health issues, particularly if they are linked to 
a negative health consequence (Bernstein, 2009).

Unfortunately, there are several barriers to effective detection of elder mistreatment in the health care setting where current approaches for identifying mistreatment rely on individuals' completion of triage processes or bedside recognition of potential abuse. These methods are inconsistent and often ineffective (Baker et al., 2016; Pickering et al., 2014). Potential challenges to providing an adequate screening and intervention program include the high acuity of conditions presenting to emergency care settings, high patient volume, high provider workload, lack of physical space to ensure privacy for such conversations, lack of provider training in screening, reporting, and addressing elder mistreatment, low provider confidence for addressing these topics, and limited resources and staffing to support such a program (Platts-Mills et al., 2020; Rosen et al., 2016). Currently, it is estimated only 1 in 24 cases of elder mistreatment become known to authorities (Storey, 2020).

Digital health tools have the potential to address many of the challenges in detecting elder mistreatment in the emergency department. These tools can be administered uniformly to patients at the point of contact with the health system for example, through a computer kiosk or tablet given to patients during waiting times - and can help "meet the person where they are" by incorporating interactive features and content that is personalized to the individual. Older adults exposed to such tools have reported improved wellbeing, empowerment, and self-efficacy (Lifshitz et al, 2016; McMellon \& Schiffman, 2002; Morris et al., 2007). The benefits of these new tools (Abujarad \& Vaca, 2015; Pickard et al., 2016) may be optimized by placing the needs of older adults in the center of the design of digital health applications and ensuring usability features that will facilitate use among older adults, who may have lower confidence using these technologies and have a higher prevalence of disabilities that make technology use more challenging (Anderson \& Perrin, 2017). Utilizing a digital health tool in the current workflow of the health care setting may address some of these barriers for detection and facilitate the screening process.

The objectives of this study were to obtain the perspectives of older adults, caregivers, and emergency care providers about screening and interventions for elder mistreatment in the emergency department, with an emphasis on the use of digital health tools. By gathering these perspectives, we aimed to inform the development of a digital health tool optimized for a tablet device to educate, screen, and facilitate reporting of elder mistreatment (the "Virtual cOaching in making Informed Choices on Elder Mistreatment Self-Dis- closure", or VOICES, program). We focused on key barriers to uptake that would need to be addressed to ensure a successful program. Incorporating the viewpoints of older adults themselves into the development of a digital health program is a critical aspect of developing a successful screening program (A. Turner et al., 2018).

\section{Methods}

\section{Study design}

This study employed a qualitative research design. We conducted three in-person focus groups with older adults from the community, caregivers for older adults, clinicians (emergency medicine doctors), and social workers who worked in the emergency department. The caregivers $(n=2)$, clinicians $(n=2)$, and social workers $(n=2)$ were included with older adults $(n=7)$ in one focus group; the remaining two focus groups were older adults only ( $n=8, n=10)$, as we wished for these voices to be the focus of the study. Focus groups were led by two moderators (EKC and FA) and attended by a note-taker (CE).

\section{Interview guide development}

We developed a semi-structured interview guide designed to identify perceptions about the process of divulging abuse, attitudes towards the emergency department as the location for screening and information, and perceptions of digital tools for screening and information. The guide was iteratively revised after multiple rounds of review and cognitive testing with several older adults and experts in geriatric care.

\section{Setting and population}

Participants were English-speaking older adults (age 60 or older) recruited through flyers and brochures posted at the Agency on Aging of South Central Connecticut (AOASCC). Inclusion criteria for these participants included the ability to communicate verbally in English along with being age 60 or older. Caregivers of older adults, and clinicians, and social workers were recruited through the assistance of the Yale Center for Clinical Investigation and direct outreach by the clinicians at the Yale New Haven Hospital main campus. Inclusion criteria for social workers and clinicians required that they be active emergency medicine social workers and doctors, respectively. Caregivers were required to be caring for community-dwelling older adults to participate. Convenience sampling was utilized for all participants. Written informed consent was obtained from all participants and data with identifiers were de-identified for post-analysis. The first focus group was held at the Yale School of Medicine in New Haven, CT, with the remaining two focus groups located at the AOASCC. Sessions were conducted in English and took approximately 70-80 minutes per session. All proce- 
Table 1. Focus group participant demographics (Older adults)

\begin{tabular}{|c|c|}
\hline Characteristic & $\begin{array}{c}\text { Older adults } \\
\text { participants }(n=24)\end{array}$ \\
\hline Age, Median (Mean) & $70(71)$ \\
\hline \multicolumn{2}{|l|}{ Gender } \\
\hline Female & $20(83 \%)$ \\
\hline Male & $4(20 \%)$ \\
\hline \multicolumn{2}{|l|}{ Marital status } \\
\hline Single/never married & $6(25 \%)$ \\
\hline Married & $9(38 \%)$ \\
\hline Divorced/Separated & $6(25 \%)$ \\
\hline Widowed & $3(13 \%)$ \\
\hline \multicolumn{2}{|l|}{ Race } \\
\hline African American/Black & $12(50 \%)$ \\
\hline Asian & $1(4 \%)$ \\
\hline White & $9(38 \%)$ \\
\hline Other & $2(8 \%)$ \\
\hline \multicolumn{2}{|l|}{ Ethnicity } \\
\hline Not Hispanic/Latino & $21(88 \%)$ \\
\hline Hispanic/Latino & $1(4 \%)$ \\
\hline Unanswered & $2(8 \%)$ \\
\hline \multicolumn{2}{|l|}{ Education } \\
\hline Less than high school & 0 \\
\hline High school degree or equivalent & $4(17 \%)$ \\
\hline Some college & $12(50 \%)$ \\
\hline Bachelor's degree & $3(13 \%)$ \\
\hline Master's degree & $4(17 \%)$ \\
\hline Doctoral degree & $1(4 \%)$ \\
\hline \multicolumn{2}{|l|}{ Income } \\
\hline Less than $\$ 30,000$ & $12(50 \%)$ \\
\hline$\$ 30,000$ to $\$ 49,999$ & $3(13 \%)$ \\
\hline$\$ 50,000$ to $\$ 69,999$ & $3(13 \%)$ \\
\hline$\$ 70,000$ to $\$ 89,999$ & $2(8 \%)$ \\
\hline$\$ 90,000$ or more/year & $3(13 \%)$ \\
\hline Refused to answer & $1(4 \%)$ \\
\hline
\end{tabular}

dures were approved by the Yale University Human Investigation Committee (IRB). Participants were provided a $\$ 30$ gift card as compensation.

\section{Data analysis}

Focus group discussions were captured on digital audio recording software, transcribed verbatim, and entered into data management software (NVivo). The study team reviewed each interview transcript and associated notes at least once before beginning analysis. Initial codes were created based on the framework of major topical headings in the interview guide. The coding structure was refined iteratively by coding transcripts, identifying additional codes through group discussion, and modifying and refining existing codes. Research team members reviewed coding categories together to ensure there was a shared understanding of the codes and to identify any needed revisions of the codebook. An integrated set of codes, consisting of all mutually agreed-upon codes, was entered into the NVIVO database with the final version of each transcript. The final coding classification scheme was applied to each transcript by two independent coders. Any coding discrepancies or ambiguities were resolved through discussion. Notes were reviewed to ensure the team understood elements such as the context of the conversation, tone, and interactions between focus group participants.
After coding all transcripts in this manner, an initial thematic framework was developed by summarizing codes by major themes and subthemes. The study team, composed of experts in elder abuse, emergency care, violence research, and qualitative methods, met collaboratively to decide on a final thematic framework and to select illustrative quotes, representing the full range of responses relevant to each theme. Differences in perceptions and data interpretation were addressed through discussion and review of transcripts and coded materials, ensuring that quotes were understood in context.

\section{Results}

We recruited a total of 31 participants, including 24 older adults, three social workers, two emergency department clinicians, and two caregivers. The characteristics of the older adult participants are provided in Table 1. Fifty percent of participants were Black/African American and 4\% Hispanic/Latino, compared to a New Haven County population that is 15\% Black/African American and 19\% Hispanic/Latino (United States Census Bureau. New Haven County, Connecticut, 2019). Among the social workers, clinicians, and caregivers, there were 5 women, the average age was 47 , and representation was $28.5 \%$ Asian, $28.5 \%$ Black or African American, and 28.5\% White.

Thematically, the focus groups provided comments about general attitudes around disclosure of elder mistreatment, responses specific to disclosure in the emergency department setting, responses specific to the use of technology to educate and screen for elder mistreatment, and recommendations for "ideal" features of a screening program in the emergency department (Tables 2-5).

\section{General perceptions about screening and disclosure}

We first solicited general attitudes about divulging elder mistreatment. Participants felt disclosure would be difficult and make older adults feel "really exposed." They expressed concerns about losing a home, support, and connection to their caregiver and loved ones. The relationship to their caregiver, with whom they often have a genuine concern and love, was also seen as a barrier to disclosure: one respondent described a situation she witnessed:

"...we had a neighbor who was being abused by her daughter who was a realtor. And she refused to share it with anyone. And this woman was very frail... And she was afraid to report because she didn't want ... she loves her daughter. She didn't want anything to happen to the daughter that would make life even harder for the daughter." (Community participant, Female)

These kinds of reservations about the negative consequences of self-reporting were the most 
Table 2. General perceptions about divulging elder mistreatment

Themes

Representative quotes

Disclosure is difficult due to pride, privacy, prior trauma

Participant 1 (Male) [FG \#1]: “I don't know. Maybe your pride. Your pride or you just don't want to talk about it. It's hard to talk about stuff like that."

Concrete benefits of withstanding abuse (financial support) are in contrast to the uncertain benefits of divulging abuse and seeking help

Participant 5 (Female) [FG \#3]: "I think it would just snowball into asking a lot of questions about my whole life. I'd feel really exposed. So it would be difficult for me."

Participant 5 (Female) [FG \#3]: "For some women ..., do they feel like loss of the home, loss of mobility, they can't help themselves, especially if that person is the person that puts the roof over their head or ... now they just don't have that. And sometimes they trade off the abuse for, "I do have a place to live.'"

Participant 15 (Female) [FG \#1]: "Yeah. What... that abused person... what happens to them? They know if they open their mouths, they gonna go home and get beat up worse to what they did. Before, they just been slapped around, now they gonna be..."

Attachment to their own home Social Worker 1 (Male) [FG \#1]: The same things happens, when, in my experience, when I may prevent disclosure meet with this particular type of patient, who presents with the same abuse; physical abuse, emotional, financial, by a child or a loved one, "I just want to go home."

Connection and loyalty to the Social Worker 3 (Female) [FG \#1]: "You also have to respect the fact. If my caregiver is the abuser prevents disclosure one who's abusing me [crosstalk]. Financially or physically. And that happens to be my daughter or my son. So, there's an emotional attachment."

Aging may come with loss of "worthiness" and sense of deserved abuse/neglect
Participant 4 (Female) [FG \#2]: "And I think also many of us when we're older and losing faculties as you say. We don't have the deep connection and the feeling of the love and warmth around us. The connections with family and friends. I think we lose a sense of worthiness, a sense of feeling important."

Participant 1 (Female) [FG \#2]: "And I think the, like you say, older, different generation of people. Back then they didn't have all this support. You know what I mean? So they're mindset is geared towards them coming up, back in their day there wasn't all that support for abuse and all this. They had to just go with it."

Participant 11 (Female) [FG \#1]: "Sometimes people are brainwashed, so to speak, and they may think that this is normal, because it's been done to them for so long."

concrete and prominent. In contrast, respondents expressed uncertainty about the potential benefits of reporting. Participants also mentioned perceptions of norms that they should just "go with [the abuse]" rather than expecting not to be mistreated (Table 2).

\section{Perceptions about the emergency department as the site of screening and disclosure of elder mistreatment}

Participants brought up a number of challenges of disclosure of elder mistreatment in the emergency department. Some felt the emergency department was not the best place for screening for mistreatment ("...first of all, it's not a very pleasant place to be" - Community participant, Female). Participants felt that this kind of conversation required to respect and a caring relationship with someone. The brief nature of the visit, in which emergency department staff are meeting a patient for the first time, rather than in the context of a longer-term relationship, was seen as a barrier to achieving sufficient trust needed to discuss something like elder mistreatment. Clinicians and social workers felt the role of hospital staff in mandatory reporting was seen as a potential barrier to disclosure.

Both community members and emergency department staff expressed uncertainty and frustration about what happens after the disclosure, the health system's ability to improve safety or to have a clear endpoint even once it knows about elder mistreatment, and acknowledged the limitations of ensuring safety beyond admitting the patient to the hospital:

Social Worker 1 (Male) [FG \#1]: "I mean, you could, but then the question becomes... At XXX Hospital, we always keep people safe, and we do a great job of that. In the scheme of managed care, we will keep this patient, but for how long?" (Social worker, Male)

Emergency department staff commented on how artificial standardized screening questions can feel to deliver, and the difficulty of identifying non-physical forms of abuse (Table 3).

\section{Responses to digital health tools for elder mis- treatment identification}

Participants were shown several examples of prototypes with illustrations and text for a digital health tool for screening elder mistreatment on a tablet device via PowerPoint (Figure 1). Each slide depicted an image of a tablet device with an illustrated concept of various aspects of the digital health tool to simulate what a user may see when looking at the screen. Specifically, participants were asked to imagine being handed a tablet device, such as an iPad, via these prototypes in the emergency department and how they might perceive that experience (Figure 1). 
Table 3. Perceptions toward screening for elder mistreatment in the emergency department

Themes Representative quotes

Participants did not perceive the Participant 11 (Female) [FG \#1]: "You can't do it [screening] in emergency. [crosstalk] not emergency department as an ideal place for screening

Participant 15 (Female) [FG \#1]: "I don't know. The home probably is the best place, but if that's where all the abuse is, and the kids are going to be in the next room, they're going to be too afraid to tell the truth...."

Caregiver 2: "Doctor's office."

Participant 15 (Female) [FG \#1]: "Well, what about their General Practitioner... They're going in for their physical and then, probably, it just going to have to be a longer time."

Participant 7 (Female) [FG \#2]: "How long is this going to be because if somebody's in a emergency room, first of all, it's not a very pleasant place to be. And then also it could be in the middle of the night."

Participant 1 (Female) [FG \#2]: "But the abuser might be in the room with you."

Mandated reporting by health professionals is viewed as a potential barrier to patient disclosure

Social Worker 2 (Male) [FG \#1]: "Well, you tell me something and then you say you're not going to tell anybody. You know that you're a mandated reporter, you have to report it to someone. Would that be something you appreciate if you're a family member disclosing abuse and I'm saying to you, 'You may not be able to take that patient home.' ... because I struggle with that as a clinician. ... honest and brutally honest is the best."

Participant 1 (Female) [FG \#2]: "Well it used to be [that elder mistreatment disclosure would occur to] your doctor. [crosstalk] But now everything has to be disclosed. You would trust your doctor with everything or anything, [crosstalk]. But now that's not the ... sometimes it's not the case because they have to report certain things that they see. It's mandated."

Disclosure is difficult due to pride, privacy, prior trauma

Participant 1 (Male) [FG \#1]: "I don't know. Maybe your pride. Your pride or you just don't want to talk about it. It's hard to talk about stuff like that."

Participant 5 (Female) [FG \#3]: “I think it would just snowball into asking a lot of questions about my whole life. I'd feel really exposed. So it would be difficult for me."

Uncertainty about the consequences of divulging abuse/neglect is a major factor in disclosure.
Participant 7 (Female) [FG \#2]: "It also can be the fear of, she's taking care of me, what if they take her away? Who's going to take care of me?"

Participant 1 (Male) [FG \#1]: "You don't know what's going to happen afterward. You tell it, and whoever's doing it might come and beat you up worse"

Participant 3 (Female) [FG \#2]: "I think maybe some of them would say, "What are they going to do about this anyway?' Why am I answering this?"

Participant 5 (Female) [FG \#3]: “... however they phrase it. I would know what they're leading up to. And I would be hesitating to answer for fear that the unknown, that something terrible is going to happen here. So maybe I need to keep that to myself." Difficult to detect non-physical ED Provider 1 (Female) [FG \#1]: "I think that's a good point too, because in a health care forms of abuse in general provider role, when it's physical abuse, we can see it, but what's really hard to tell is when there is some form of emotional abuse"

\section{Standardized screening} language can feel stilted
ED Provider 2 (Female) [FG \#1]: "But I use very concrete examples. And I've had success with that, because a lot of times I feel like, especially in emergency departments, we're trying to move quickly, there's routine checklist, 'Do you feel safe at home? Do you have this, do you have that?' That's not a real conversation, I don't think."
While participants acknowledged potential technical challenges to digital health tools, they also readily identified advantages of utilizing technology for this population, including the familiarity, consistency, ability to go at one's own pace, and potential for asynchronous involvement.

"And then when you're sharing something that's so deep and personal and you see if the person is distracted or looks away or looks at the time, it's hard to keep going. Whereas, an iPad, you can take your time, you can share it." (Community

\section{participant, Female)}

Participants discussed feelings with the prototype itself and expressed an overall preference for viewing educational videos with illustrations rather than clips involving real people. They felt that the tool needed to be brief, both in content and for the length of animated educational videos. Participants preferred for text on the screen to be spoken aloud with a female narrator and headphones for privacy and emphasized the 


\section{Tap on a category to learn more.}
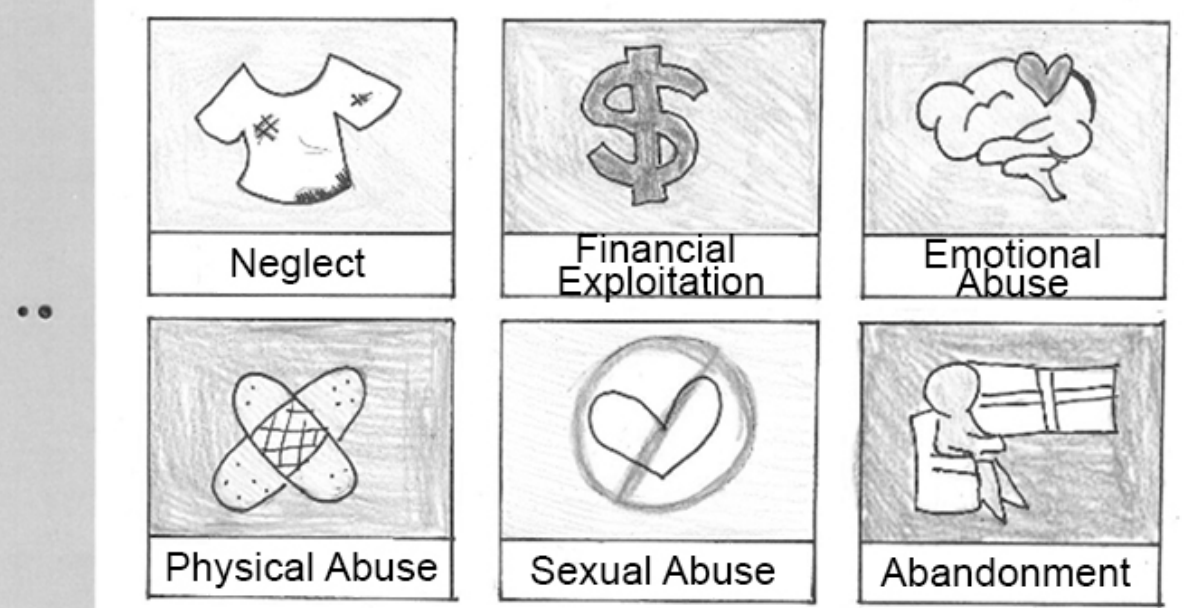

[10]

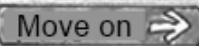

Figure 1. Illustrated prototype of a concept for a digital elder mistreatment screening tool. This screen depicts buttons that can be touched by the user to view videos relating to specific types of mistreatment

need for the tool to be user-friendly, in particular with older adults who had never utilized a tablet device before. Some participants felt that the tool should consist of "Yes" and "No" binarystyle questions, rather than responding to questions with an open-ended typing format (Table 4).

\section{Recommendations for successful screening and interventions for elder mistreatment}

Participants identified a number of desired aspects of screening in the emergency department, often specifically in response to the challenges and concerns raised about disclosure in general in the ED, with regards to digital health tools. Participants recommended being clear about terms and definitions to assist those who have not recognized that abuse is happening. They recommended ensuring privacy and conveying empathy and connection as much as possible in a brief interaction, being sure to avoid making the patient feel rushed. Empathy for the abusive person, who often may be a family member that the patient has considerable loyalty to and affection for, was felt to be potentially helpful in conversation with a patient.

"It's not just, 'That person is a bad person.' That person may be overwhelmed, maybe that person have their own family and they're trying to help their mother, and they're just stressed to the max." (Social worker, Female)
Participants also felt it was important to be as clear and transparent as possible about the implications of disclosure, including addressing what happens immediately after disclosure, confidentiality, the involvement of law enforcement, and potential outcomes of reporting. Participants were overall positive about leveraging technology but felt it was important to address concerns about technical challenges and preferences for a human conversation by providing technical assistance and offering the option to interact with a person as well (Table 5).

\section{Discussion}

Systematic identification of elder mistreatment remains a high priority needs across healthcare settings. Computer or Web-based programs have numerous advantages in achieving this goal. They provide a sense of anonymity and privacy, which may increase reporting of factors impacting health, including sensitive topics like substance use, sexual behaviors, and victimization (Rhodes et al., 2006; C. F. Turner et al., 1998). They bypass the difficulties of training and maintaining the knowledge and skills of a massive workforce, requiring little direct clinician involvement. They can be adapted to be culturally and linguistically specific and audio capabilities allow interventions to engage low-literacy individuals. They can deliver assessments and, 
Table 4. Attitudes toward screening for elder mistreatment through digital health tools

$\begin{array}{ll}\text { Themes Representative quotes } & \text { Rus }\end{array}$

The technical aspect of computer screening may be challenging for some elders
Participant 15 (Female) [FG \#1]: "Most things, like my mother never... She saw our

computers but never she tried it, she didn't want to learn it. I have a 93-year old uncle right now that I'm kinda helping take care of. He don't wanna have nothing to do with a computer. What do you do about that?"

Participant 7 (Female) [FG \#3]: "If you're abused an you're answering this, this is ... Who's going to read this? And if I'm not... savvy with computers and stuff, it's harder on me."

ED Provider 1 (Female) [FG \#1]: "I think you would still have to have someone on the ground, though. So, if I think of my mom, she would be, like, "How do I do this? I don't know. What's this thing say? What is this pop-up?"

Many advantages to technology, Participant 7 (Female) [FG \#3]: “I'm going to tell you ... I won't go into detail, but I know including ability to take one's people who stay in the house where there's mental, physical abuse. The computer is what time, consistent attention, and ability to refer back later they're on all the time because it's something to concentrate, something to do, they have nobody else to talk to, and that gives them comfort."

Participant 9 (Female) [FG \#3]: “If I'm really upset, I don't know. I think it's [technologybased screening] a good idea. I think it's a good concept. If I'm really upset, I don't know. But then I don't think anything would work if I'm anxiety ridden"

Participant 6 (Female) [FG \#3]: "Maybe at the end something that says if you don't think you're ready to do something about it now, where you can go once you're discharged."

Technology viewed as an adjunct to human processes, rather than a complete replacement

Desired features of a computerbased screening digital health tool
ED provider 1 (Female) [FG \#1]: "I think you would still have to have someone on the ground, though. So, if I think of my mom, she would be, like, "How do I do this? I don't know. What's this thing say? What is this pop-up?"

\footnotetext{
Participant 1 (Female) [FG \#2]: "So I might be more inclined to answer on that iPad, because then I don't have... interference of someone interacting with me, their opinion... I think an iPad would be really good."
}
Participant 1 (Female) [FG \#2]: "I think... video is a good idea. And I think it can be short. Very quick."

Participant 3 (Female) [FG \#3]: “I agree [with Participant 11] it has to be a female voice."

Participant 3 (Female) [FG \#3]: “...it has to be colorful."

Participant 5 (Female) [FG \#3]: "I think the cartoons [for video] as well. ...it takes the edge off of [the topic of mistreatment], the cartoons."

Participant 10 (Male) [FG \#1]: "I think a cartoon would be more relaxing."

Participant 7 [FG \#3]: “There's a commercial...on TV where they're dialing 911, and they're nervous, and they're crying... that upsets me so much. ...something that realistic, I don't think I could handle that."

Participant 11 (Female) [FG \#1]: "Not too many words."

Participant 11 (Female) [FG \#1]: "It has to be user-friendly for [older adults] because they're from a different era."

based on the results, provide immediate, individualized feedback and recommendations for change. Participants in our study cited many of these aspects as potential benefits of digital screening tools for elder mistreatment in the emergency department.

Digital platforms minimize the bias that can arise in interpersonal relationships between the provider and patient. Such platforms can scale up and allow us to screen and/or intervene in large numbers of older adults with minimal cost and effort. Also, they can be widely disseminated while maintaining treatment fidelity across clinical sites. Americans, including older adults, report increasing comfort with the use of technology (Anderson \& Perrin, 2017), including the Internet and popular social media sites. Most emergency department patients (including older adults) use computers (91\%) and access the Internet $(71 \%)$ and the vast majority feel comfortable receiving technology-based health information (Ranney et al., 2012).

Our study corroborated several themes from prior literature, including general barriers to disclosure such as fear, vulnerability, and loss of home, social supports and relationships, and emer- 
Table 5. Recommendations for successful screening and interventions for elder mistreatment

$\begin{array}{ll}\text { Themes } & \text { Representative quotes }\end{array}$

Clarity in definitions, framing Caregiver 2 (Female) [FG \#1]: Do you think they all understand what neglect is? ... if you're is important so that abuse is recognized as such

in a situation and it's getting worse and worse and worse, you sort of lose your perspective of, 'Am I being neglected, or is this what I deserve, or is this...' A piece that's really important, but I don't know if they all understand neglect."

ED Provider 1 (Female) [FG \#1]: "How do we frame it in a way that we can really address it to get the patients to open up, because the patient might not even realize that's abuse."

Important for providers to set ED Provider 1 (Female) [FG \#1]: "Really, sitting down, nice, quiet environment, not in the the scene for disclosure. hallway. Making sure the patient is comfortable in terms of food, water, music, whatnot, and Screening needs to occur in a saying, 'Hey, I'd like broach a difficult subject with you. I know you might not want to share setting with privacy and confidentiality, including distance from abuser right now, but I feel like we have a relationship. Can I talk to you about some of the concerns I'm having?'"

Participant 11 (Female) [FG \#3]: "But she said, 'Okay, you have a lot of injuries. Is there anything you'd like to tell me. Just tell me exactly how it happened...' And a lot of nice little questions... and she was taking little notes on the [crosstalk]. I thought her approach was lovely and her personality or whatever. She was approachable."

Participant 7 (Female) [FG \#3]: "When you're sitting next to somebody who's that evasive or whatever, but you're in, usually, a small chair. It's not comfortable. You need to be in a separate area and be able to have it like that and be able to touch it or key it and whatever you want."

Participant 7 (Female) [FG \#3]: "And I think if it's given ... that the person is going to have some privacy to do it. There's nobody else in the room."

Taking time to convey empathy and make a connection is a key step.

Participant 6 (Female) [FG \#3]: "They came only just to speak with you and be warm and make eye contact and just general ... you know ... instead of they're pulling you over on the stretcher and, 'Oh, by the way. Are you..' I mean, 'I need to ask you,' or 'I have to ask you this question.'"

Social Worker 3 (Female) [FG \#1]: "But you've got to put in that time, whether it's 15 solid minutes or half an hour. Whatever time, it's you and me and setting that empathetic stage gives them the opportunity to talk with you."

Participant 3 (Female) [FG \#2]: “Concerned and compassionate. Not making me [crosstalk] feel you don't have the time and you're hurried."

Demonstrating empathy for the Participant 4 (Female) [FG \#2]: "Well I would say, it doesn't mean you don't love them. It abuser and framing interventions as help for the whole family may be helpful.

doesn't mean that you're a bad parent or loved one. It's help to both of you. You'll both grow through this if you permit someone to enter your world and help you through this hard time."

Social Worker 3 (Female) [FG \#1]: It's not just, "That person is a bad person." That person may be overwhelmed, maybe that person have their own family and they're trying to help their mother, and they're just stressed to the max.

Need to provide up-front information about what happens after a disclosure, including confidentiality and safety measures in place
Participant 3 (Female) [FG \#3]: "if they're informed from the beginning that it's confidential ... what the outcomes may be, what types of help are available if that is their situation, that there are police officers there in the emergency room. And it think people feel a little bit better about speaking up."

Participant 6 (Female) [FG \#3]: "Because what are you going to do with it afterwards? It goes back to explaining to the person how it works or how the help comes."

Consider a mix of in-person assistance and computer screening, or choice between them
Participant 3 (Female) [FG \#3]: "I would let them choose. ... with a brief conversation like with ...what to do...And that person has the option to play the video."

Participant 6 (Female) [FG \#3]: "Maybe if whoever gave it to you can go back and check to see how you were doing with it, if you had any more questions about it. Are you able to fill it out okay? Would you rather speak to somebody than to fill that out?" gency department-specific concerns, including skepticism whether conversations around mistreatment can occur in the context of acute health problems, potential lack of privacy, and a caring relationship that is new and temporary. Reassurances about these concerns must be built into the program content and/or the introduction and delivery of the program by clinical staff. Any intervention protocol will have to guarantee privacy for the participant (e.g., by being administered once a patient is in a private room, rather than in registration, triage, or waiting room), build in explicit mitigation of concerns around safety and consequences of divulging abuse, include details about what happens after disclosure, and provide examples of the resources and 
supports available to those experiencing elder mistreatment. While the use of digital screening tools, in general, have established acceptability and feasibility in the ED, a tool for elder mistreatment incorporating all these elements will need to be established specifically for elder abuse.

Participants overall felt there were many benefits of using computers to screen. However, they acknowledged that some older adults (referencing their own parents more than themselves) may not be completely comfortable engaging with technology and felt the process of screening for mistreatment could likely not be fully automated but require some human support. Earlier work demonstrated efficacy in addressing violence in younger populations through computer-only interventions (Cunningham et al., 2013); older adults may require more of a hybrid model. While there were concerns associated with the use of digital health technology for computerbased screening, the literature indicates that further exploration of computer-based interventions may benefit users overall.

A dominant theme of the focus groups was the uncertainty of any benefits of disclosure, a major concern for not only the community members themselves but for the hospital caregivers (clinicians and social workers), who conveyed frustration with the limits of intervention once mistreatment is identified. This kind of systembased, complex, and multifactorial barrier to successfully addressing mistreatment represents a fixed parameter to the success of any screening program; although programs can provide education and support, ultimately, staff in the emergency department must perform tasks that are not automatable at this time, including verifying abuse, providing direct support, meeting reporting requirements, and determining immediate care needs. Although there are clear, addressable gaps in the identification of mistreatment among older adults, our study emphasized that the impact of improved screening must occur in concert with other improvements in the care of this population to clarify the risk-to-benefit ratio.

Participants also suggested incorporating empathy for the abuser, pointing out that this person may still be a beloved family member such as a child who the patient loves and may feel protective towards. More generally, participants seemed to wish for options that did not disrupt their living situation and relationships. From a clinical perspective incorporating this desire may be challenging - particularly with mandatory reporting requirements - and the instinct may be to separate patients with mistreatment from the relationship. However, pragmatic screening and interventions may also need to "meet patients where they are," recognize nuance and complexity of patients' desires in balance with absolute health and safety considerations.

Finally, participants recommended a number of technical elements for the intervention, including visual and graphical preferences, many of which underscored the specific needs of older adults, including accommodations for those with visual and/ or hearing impairment or low skill with technology. These preliminary perceptions helped guide the framework of the tool's initial development. Following these perceptions and the initial development of the tool, a study will be conducted to test the usability and acceptability of the tool.

\section{Conclusion}

Our study suggested that digital health platforms have the potential to overcome the numerous concerns related to screening for elder mistreatment in general and in the emergency care setting. Interview themes supported several key adaptations to our original concept, to the digital program itself, and to the way it is administered and supported. These adaptations will allow an initial version of the digital tool for testing within the emergency department.

Our study did have a number of limitations. We did not specifically identify a group of older adults who were known to be experiencing mistreatment or to have experienced it in the past, so input may be conjecture rather than from direct experiences. However, given the high prevalence of elder mistreatment, we did assume a high likelihood of direct or witnessed experiences, and the focus group discussions supported this assumption. Because we wished to focus primarily on older adults from the community to support the design of digital tools for screening this population, we only included a small sample of clinicians, caregivers, and social workers. These participants were included in one of the three focus groups, along with community participants, rather than in a focus group or groups dedicated to them. The interview guides were also designed to be inclusive of these participants, but not focused on their experiences, given the purposes of this study. Additional work is needed to represent the views and experiences of this group in more depth.

Finally, we lacked some important representation in the study. All of the participants had at least a high school degree; this relatively highliteracy population may have had a more positive attitude toward technology-based screening than the average emergency department population. Fifty percent of community older adult participants were Black/African American, but only $4 \%$ were Asian, and $8 \%$ other race; only 
4\% were Hispanic/Latino. We did not include those with a primary language other than English. Caregivers and hospital staff were under-represented and as a focus of a future study would need to be recruited in greater numbers. Here, our focus was on the older community members. Additional research to ensure culturally specific themes for program tailoring is needed to fully understand the perceptions about elder mistreatment screening in the emergency department and via digital platforms, and to ensure broader generalizability of the content, format, and functionality of screening programs designed from this exploratory work.

\section{Acknowledgements}

Dr. Gill is supported by the Yale Claude D. Pepper Older Americans Independence Center (P30AG021342).

\section{Funding}

This work was supported by the National Institute on Aging of the National Institutes of Health (R01AG060084).

\section{References}

Abujarad, F., \& Vaca, F. (2015). mHealth Tool for Alcohol Use Disorders Among Latinos in Emergency Department. Proceedings of the International Symposium on Human Factors and Ergonomics in Health Care, 4(1), 12-19.

Acierno, R., Hernandez, M. A., Amstadter, A. B., Resnick, H. S., Steve, K., Muzzy, W., \& Kilpatrick, D. G. (2010). Prevalence and Correlates of Emotional, Physical, Sexual, and Financial Abuse and Potential Neglect in the United States: The National Elder Mistreatment Study. American Journal of Public Health, 100(2), 292-297.

Anderson, M., \& Perrin, A. (2017). Tech Adoption Climbs Among Older Adults. Pew Research Center. Accessed September 18, 2020.

Baker, P. R., Francis, D. P., Hairi, N. N., Othman, S., \& Choo, W. Y. (2016). Interventions for preventing abuse in the elderly. Cochrane Database of Systematic Reviews, (8).

Bernstein, S. L. (2009). The clinical impact of health behaviors on emergency department visits. Academic Emergency Medicine, 16(11), 1054-1059. https:// doi.org/10.1111/j.1553-2712.2009.00564.x

Centers for Disease Control and Prevention. (2016). Understanding Elder Abuse. Accessed October 30, 2020. (n.d.). https:/www.cdc.gov/violenceprevention/pdf/em-factsheet-a.pdf

Cunningham, R., Whiteside, L., Chermack, S., Zimmerman, M. A., Shope, J. T., Bingham, C. R., Blow, F. C., \& Walton, M. A. (2013). Dating violence: outcomes following a brief motivational interviewing intervention among at-risk adolescents in an urban emergency department. Acad Emerg Med, 20(6), 562-569.

Lifshitz, R., Nimrod, G., \& Bachner, Y. G. (2016). Internet use and well-being in later life: a functional approach. Aging \& Mental Health, 22(1), 85-91. https://doi.org/10.1080/13607863.2016.1232370

McMellon, C., \& Schiffman, L. (2002). Cybersenior Empowerment: How Some Older Individuals Are Taking Control of Their Lives. Journal of Applied Gerontology, 21(2), 157-175.

Morris, A., Goodman-Deane, J., \& Brading, H. (2007). Internet use and non-use: Views of older users. Universal Access in the Information Society, 6, 43-57.

Pickard, M., Roster, C., \& Chen, Y. (2016). Revealing sensitive information in personal interviews: Is selfdisclosure easier with humans or avatars and under what conditions? Computers in Human Behavior, 65, 23-30.

Pickering, Carolyn E. Ziminski, and Veronica F. Rempusheski. (2014). Examining barriers to self-reporting of elder physical abuse in community-dwelling older adults. Geriatric Nursing 35.2, 120-125.

Platts-Mills, T., Hurka-Richardson, K., Shams, R., Aylward, A., Dayaa, J. A., Manning, M., Mosqueda, L., \& et al. (2020). Multicenter Validation of an Emergency Department-Based Screening Tool to Identify Elder Abuse. Ann Emerg Med, 76(3), 280-290.

Ranney, M. L., Choo, E. K., Wang, Y., Baum, A., Clark, M. A., \& Mello, M. J. (2012). Emergency department patients' preferences for technology-based behavioral interventions. Annals of Emergency Medicine, 60(2). https://doi.org/10.1016/j.annemergmed.2012.02.026

Rhodes, K., Drum, M., Anliker, E., Frankel, R., Howes, D., \& Levinson, W. (2006). Lowering the threshold for discussions of domestic violence: a randomized controlled trial of computer screening. Arch Intern Med, 166(10), 1107-1114.

Rosay, A. B., \& Mulford, C. F. (2017). Prevalence estimates and correlates of elder abuse in the United States: The national intimate partner and sexual violence survey. Journal of elder abuse \& neglect, 29(1), 1-14.

Rosen, T., Bloemen, E., LoFaso, V., Clark, S., Flomenbaum, N., \& Lachs, M. (2016). Emergency Department Presentations for Injuries in Older Adults Independently Known to be Victims of Elder Abuse. The Journal of Emergency Medicine, 50(3), 518-526.

Storey, J. E. (2020). Risk factors for elder abuse and neglect: A review of the literature. Aggression and Violent Behavior, 50, 101339.

Turner, A., Osterhage, K., Taylor, J., Hartzler, A., \& Demiris, G. (2018). A Closer Look at Health Information Seeking by Older Adults and Involved Family and Friends: Design Considerations for Health Information Technologies. AMIA Annu Symp Proc, 2018, 1036-1045.

Turner, C. F., Ku, L., Rogers, S. M., Lindberg, L. D., Pleck, J. H., \& Sonenstein, F. L. (1998). Adolescent sexual behavior, drug use, and violence: increased reporting with computer survey technology. Science (New York, N.Y.), 280(5365), 867-873. http://www. ncbi.nlm.nih.gov/pubmed/9572724

United States Census Bureau. 65 and Older Population Grows Rapidly as Baby Boomers Age. Accessed Sept 30, 2020. (2020). https://www.census.gov/ newsroom/press-releases/2020/65-older-population-grows.html 


\section{Digital screening tools for elder mistreatment}

United States Census Bureau. New Haven County, Connecticut. Accessed October 1, 2020. (2019). https://www.census.gov/quickfacts/newhavencountyconnecticut

United States Census Bureau. The U.S. Joins Other Countries With Large Aging Populations. Accessed
Sept 30, 2020. (2018). https://www.census.gov/library/stories/2018/03/graying-america.html

Yon, Y., Mikton, C. R., Gassoumis, Z. D., \& Wilber, K. H. (2017). Elder abuse prevalence in community settings: a systematic review and meta-analysis. The Lancet Global Health, 5(2), e147-e156. 